Pacific Journal of Mathematics

DIFFERENTIABLE APPROXIMATIONS TO HOMOTOP 


\title{
DIFFERENTIABLE APPROXIMATIONS TO HOMOTOPY RESOLUTIONS AND FRAMED COBORDISM
}

\author{
DONALD W. KAHN
}

\begin{abstract}
The determination of homotopy groups of spheres remains a central problem in algebraic topology. The various methods for addressing this problem cover some considerable ground, from intricate algebra to the structure of manifolds. Our general purpose here is to show that the gap between some of these methods can in fact be closed, and that one may find geometric structures (manifolds) which reflect the filtrations arising from algebraic methods.
\end{abstract}

To be specific, the different methods for studying homotopy groups of spheres include the construction of homotopy resolutions, [9], the Adams spectral sequence, [1], and its variants, and the theory of framed cobordism, [8], which is historically the first general method. Naturally, there are now many extensions of these methods, and a great many results have been obtained. But even in the area where the groups are now well-known, the relationship between the different methods is often not clear. For example, given a specific known element in $\pi_{i}\left(S^{j}\right)$, in the stable range, one does not always know specific manifolds which represent the element in framed cobordism, or specific cohomology operations which detect the element, not to mention a finitely-computable procedure for determining when a given map $f: S^{i} \rightarrow S^{j}$ would represent such an element. An early attempt to analyze the relationship between the methods of homotopy resolutions and the Adams spectral sequence is the paper of H. Gershenson [4]. Since that time, there has been some work on analyzing what sort of manifolds, for example Lie groups, can carry framings which represent certain classes in homotopy groups of spheres. See, for example [2]. Recent results of E. Ossa [7] are remarkable in terms of showing how relatively little can be carried invariantly on Lie groups.

The present paper originates in my idea that it should be possible, at least in theory, to bridge the gap between framed cobordism and the theory of homotopy resolutions (Postnikov towers). Specifically, one should be able to build a "filtration" in framed cobordism, which is some sort of reflection of the basic homotopy resolution of the space. This is roughly the content of Theorem 2 below. The naive idea behind this is to 
effectuate a homotopy resolution in the category of differentiable manifolds without boundary. But a little reflection about the cohomology of Eilenberg-MacLane spaces and Poincare duality will show that it is a rare event indeed when an Eilenberg-MacLane space is such a manifold. Therefore, one looks for manifolds and maps, which approximate in the sense of $k$-type, a given, finite piece of a homotopy resolution. That this can be done in all reasonable cases is our Theorem 1 . We also indicate, in passing, how one might interpret the $k$-invariant in this differentiable setting. Some examples and applications follow Theorem 2 . We begin with some preliminary material and two lemmas.

It is my primary intention to try to stimulate interest in this area. There are only a few cases, where explicit computation appears to be practical at this time.

We work in the category of 1-connected spaces with base point. Maps (continuous) and homotopies respect the base points, although we usually omit them from the notation. All our spaces have the homotopy-type of a $\mathrm{CW}$-complex, with finitely-generated integral homology in each dimension. For such a space $X$, a homotopy resolution for $X$ (see [5]) will mean a family of spaces and maps

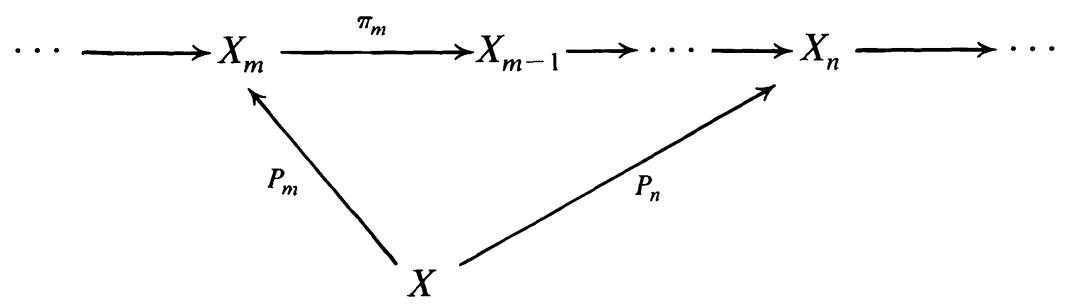

so that

(1) $\pi_{n} \cdot P_{n}=P_{n-1}$

(2) $\pi_{n}$ is an $(n-1)$-equivalence; $P_{k}$ is a $k$-equivalence.

(3) $\pi_{j}\left(X_{k}\right)=0$, if $j>k$.

If $X$ is $(n-1)$-connected, then $X_{n}$ is clearly a $K\left(\pi_{n}(X), n\right)$ space. Note that we do not assume that the $\pi_{m}$ are principal fibrations, with fibre $K\left(\pi_{m}(X), m\right)$, because there is no ready analogue for them in the world of compact, differentiable manifolds.

The literature is not consistent about what is a $k$-equivalence. Some authors want a map $f: X \rightarrow Y$, inducing isomorphisms in homotopy through dimension $k$; others will settle for a map defined on some skeleton. To make sense of a $k$-equivalence, as distinct from a homotopyequivalence, I require that there be a map $f: X^{(l)} \rightarrow Y$, where $l>k$, inducing isomorphisms on homotopy through dimension $k$. 
We note, also, that the main theorems of this paper will be valid for any suitable equivalence. In this generality, the proofs are rather more difficult than merely taking skeleta and suitable smooth neighborhoods.

LEMMA 1. Given a space $X$ and $k>1$. Then there is a compact, differentiable manifold, without boundary, say $M$, having the $k$-homotopy-type of $X$. (That is there is an $f: X^{(l)} \rightarrow M$, from the l-skeleton of $X, l>k+1$, inducing isomorphisms in homotopy through dimension $k$ (and an epimorphism in dimension $k+1)$ ). We do not notate the generally large dimension of $M$.

Proof. It is well-known (see, for example, [3]) that if $K$ is a finite subcomplex of the sphere $S^{n}$, then there is a compact, differentiable $\left(C^{r}\right.$, $r \geq 1$ ) manifold with boundary, say $N$, with $K \subseteq N \subseteq S^{n}$, so that the inclusion $K \subset N$ is a homotopy-equivalence. If $n$ is much larger than the dimension of $K$, we may assume $S^{n}-N$ is highly connected. Using the Mayer-Vietoris sequence of $\left(S^{n}, N, \overline{S^{n}-N}\right)$, with $N \cap\left(\overline{S^{n}-N}\right)=\partial N$, we see that the inclusion $\partial N \subseteq N$ induces isomorphisms on integral homology groups through as large a range of dimensions as we wish, depending on the choice of $n$.

Set $M$ to be the double of $N$, gluing by the identity map along the boundary. Writing $N_{1}$ and $N_{2}$ for the 2 copies of $N$ in $M$, we have $N_{1} \cup N_{2}=M, N_{1} \cap N_{2}=\partial N$. By the above remarks, we may assume that the inclusion $\partial N \rightarrow N$ induces an isomorphism on homology through a large range of dimensions, so that it follows that in such a large range of dimensions, the Mayer-Vietoris sequence,

$$
H_{*}(\partial N) \rightarrow H_{*}\left(N_{1}\right) \oplus H_{*}\left(N_{2}\right) \rightarrow H_{*}(M),
$$

is a split sequence, and thus, for such a range, the inclusion of $N_{i}$ in $M$ must also induce an isomorphism in homology.

If we let $K$ be a suitably large skeleton of $X$, then the lemma is clear.

We note that in special cases, there are often better approximations than those given by this lemma.

LEMMA 2. Let $P: X \rightarrow X_{m}$ be any (continuous, base-point preserving) map, where we assume that $\pi_{j}\left(X_{m}\right)=0$, if $j>m$. Let $k$ be an integer, $k>m$. Then there are differentiable manifolds $M$ and $Q$, which have the 
$k$-homotopy-type of $X$ and $X_{m}$ (resp.), given by maps $f_{M}: X^{(l)} \rightarrow M$ and $f_{Q}$ : $X_{m}^{\left(l^{\prime}\right)} \rightarrow Q$, with $1<l<l^{\prime}$. In addition, there is a map $\tilde{P}$ for which

$$
\begin{array}{ccc}
X^{(l)} & \stackrel{P}{\rightarrow} & X_{m}^{\left(l^{\prime}\right)} \\
\downarrow f_{M} & & \downarrow f_{Q} \\
M & \stackrel{\tilde{P}}{\rightarrow} & Q
\end{array}
$$

is homotopy-commutative. $\tilde{P}$ may be taken to be differentiable.

We also note that $f_{M}$ and $f_{Q}$ may be chosen to be any equivalences through the correct range of dimensions.

Proof. Using Lemma 1, we easily construct a partial diagram

$$
\begin{array}{ccc}
X^{(l)} & \stackrel{P}{\rightarrow} & X_{m}^{\left(l^{\prime}\right)} \\
\downarrow f_{M} & & \downarrow f_{Q} \\
M & & Q
\end{array}
$$

where the vertical maps are $k$-homotopy equivalences, for any suitable $k>1$ that we wish. Up to homotopy-type, we may then also regard $M$ as containing $X^{(l)}$ as a cellular subcomplex, where all remaining cells of $M$, besides those of $X^{(l)}$, have dimension bigger than $k$. But on the other hand, $f_{Q}$ may be chosen to be a homotopy equivalence through a range exceeding the dimension of the cellular version of $M$, as constructed above.

But then, the obstructions to extending the map $f_{Q} \cdot P: X^{(l)} \rightarrow Q$ to a map $M \rightarrow Q$ will lie in

$$
H^{j}\left(M, X^{(l)} ; \pi_{j-1}(Q)\right)
$$

for $k<j \leq \operatorname{dim}(M)$. Since $j>k>m, j-1>m$, so $\pi_{j-1}\left(X_{m}\right)=0$ in the range at hand. Because $\pi_{j-1}\left(X_{m}\right) \approx \pi_{j-1}(Q)$ through the dimension of $M$, all groups containing these obstructions vanish, yielding the extension.

Replacing $M$ by the original differentiable manifold, rather than the cell complex, and taking a differentiable approximation yields the desired $\tilde{P}$.

REMARK. Lemma 2 is a "stable" version of a differentiable approximation in the sense that we require $k>m$.

Our first theorem is then the following: 
THEOREM 1. Let

$$
X \stackrel{P_{m}}{\rightarrow} X_{m} \stackrel{\pi_{m}}{\rightarrow} \cdots \stackrel{\pi_{n+1}}{\rightarrow} X_{n}
$$

be a finite homotopy resolution in our category of spaces. Let $k>m$. Then there is a sequence of differentiable manifolds, $M, Q_{m}, Q_{m-1}, \ldots, Q_{n}$, and maps $f: X^{(l)} \rightarrow M$ and $f_{i}: X_{i}^{\left(l_{i}\right)} \rightarrow Q_{i}, n \leq i \leq m$, for suitable integers $l$ and $l_{i}$ all greater than $k$, which are $k$-equivalences, and differentiable maps $\tilde{P}_{m}$ and $\tilde{\pi}_{i}$, so that the following diagram is homotopy commutative:

$$
\begin{array}{ccccccc}
X^{(l)} & \stackrel{P_{m}}{\rightarrow} & X_{m}^{\left(l_{m}\right)} & \stackrel{\pi_{m}}{\rightarrow} & \ldots & \stackrel{\pi_{n+1}}{\rightarrow} & X_{n}^{\left(l_{n}\right)} \\
\downarrow f & & \downarrow f_{m} & & & & \downarrow f_{n} \\
M & \stackrel{\tilde{P}_{m}}{\rightarrow} & Q_{m} & \stackrel{\tilde{\pi}_{m}}{\rightarrow} & \ldots & \stackrel{\tilde{\pi}_{n+1}}{\rightarrow} & Q_{n}
\end{array}
$$

In addition, one may assume

$$
\operatorname{dim} M>\operatorname{dim} Q_{m}>\cdots>\operatorname{dim} Q_{n} .
$$

Within the ranges of dimensions required in the proof, absolutely any choice of $k$-equivalence, for $f$ and the $f_{i}$ is permissible here.

Proof. By Lemma 2, one may easily deduce the theorem (apart from the final sentences) for a two stage homotopy resolution $X_{n+1} \rightarrow X_{n}$. If we have a 3-stage resolution $X_{n+2} \rightarrow X_{n+1} \rightarrow X_{n}$, then using Lemma 1, we may easily construct

$$
\begin{array}{ccccc}
X_{n+2}^{\left(l_{n+2}\right)} & \stackrel{\pi_{n+2}}{\rightarrow} & X_{n+1}^{\left(l_{n+1}\right)} & \stackrel{\pi_{n+1}}{\rightarrow} & X_{n}^{\left(l_{n}\right)} \\
\downarrow f_{n+2} & & \downarrow f_{n+1} & & \downarrow f_{n} \\
Q_{n+2} & & Q_{n+1} & \stackrel{\pi_{n+1}}{\rightarrow} & Q_{n}
\end{array}
$$

for suitable integers $l_{n+2}, l_{n+1}$, and $l_{n}$. Referring to the proof of Lemma 2, we may construct a horizontal map in the lower-left hand corner, $\tilde{\pi}_{n+2}$ : $Q_{n+2} \rightarrow Q_{n+1}$, provided $f_{n+1}$ is a homotopy equivalence in dimensions up to $\operatorname{dim}\left(Q_{n+2}\right)$. But the dimensions $l_{n+1}$ and $l_{n}$ may be chosen as large as we wish, as well as the dimension through which $f_{n+1}$ and $f_{n}$ are homotopy equivalences (Lemmas 1 and 2). Therefore, we change $Q_{n}, Q_{n+1}, f_{n}$, and $f_{n+1}$-if necessary - to assure that $f_{n+1}$ is a homotopy-equivalence, through a sufficiently large dimension, and the map $\tilde{\pi}_{n+2}$ may then be constructed.

It is clear that this process may be repeated for any finite homotopy resolution, completing the existence of the diagram in the Theorem. To 
arrange matters so that the dimensions are increasing, preserving the rest of the Theorem, one simply selects suitable family of spheres, of increasing dimensions, all larger than $k$, replaces the manifolds with their Cartesian products with the spheres, and the maps such as $\tilde{\pi}_{l}$ by the product of the original $\tilde{\pi}_{i}$ with a trivial map between the spheres.

Before passing to framed cobordism, we wish to briefly indicate how the $k$-invariants of a Postnikov system may be described in the framework of differentiable approximations. In other words, we wish to analyze the $k$-invariant, modulo torsion, in terms of differential forms. Consider a stage of a Postnikov tower, that is a principal fibration

$$
K(G, n) \rightarrow X_{n} \stackrel{\pi}{\rightarrow} X_{n-1} .
$$

Let $\phi: X_{n-1} \rightarrow C \pi$ be the usual map of $X_{n-1}$ to the mapping cone of $\pi$. Following Lemma 2, let $\tilde{\phi}: Q \rightarrow M$ be a differentiable approximation to $\phi$ up to $k$-homotopy type, with $k$ large.

We may easily assume that we have embedded spheres, say $S_{i}^{n+1}$, in $M$, representing generators of the free summand of $\pi_{n+1}(M)$. (Note that $M$ is $n$-connected). Suppose $\omega$ is a closed $(n+1)$-form on $M$, whose restriction to $S_{i}^{n+1}$ represents the dual to a generator in real homology. Then, the differential form $(\tilde{\phi})^{*}(\omega)$ represents the restriction of the $k$-invariant, $k^{n+1}$, to that summand in cohomology, corresponding in the coefficients to the generator represented by $S_{l}^{n+1}$, up to a possible non-zero real multiple.

We now wish to exhibit a filtration in framed cobordism, arising from a homotopy resolution. Recall that if $g: S^{p} \rightarrow S^{q}$, then the classical construction of Pontrjagin replaces $g$ by a differentiable map, and then takes the inverse image of a regular value, say $M=g^{-1}\left(x_{0}\right)$, yielding a framed manifold in $S^{p}$ (see [8]).

THEOREM 2. Given a (stable) homotopy class, represented by g: $S^{p} \rightarrow S^{n}$, and a finite homotopy resolution

$$
S^{n} \stackrel{P_{m}}{\rightarrow} X_{m} \stackrel{\pi_{m}}{\rightarrow} \cdots \rightarrow X_{n},
$$

then

(1) There is a compact (without boundary) manifold $V$, having the same $k$-homotopy-type as $S^{p}, k>p$, with $S^{p}$ a submanifold of $V$,

(2) There is associated to our resolution a decreasing family of framed submanifolds of $V$, called $W_{i}$ and $W$,

$$
V \supseteq W_{n} \supseteq W_{n+1} \supseteq \cdots \supseteq W_{m} \supseteq W,
$$


and

(3) $W \cap S^{p}$ is a framed submanifold of $S^{p}$ which represents $\{g\}$ in framed cobordism.

Proof. Up to $k$-equivalence, we may replace the resolution by

$$
M \stackrel{\tilde{P}_{m}}{\rightarrow} Q_{m} \stackrel{\tilde{\pi}_{m}}{\rightarrow} \cdots \rightarrow Q_{n} \quad\left(\tilde{P}_{j}=\tilde{\pi}_{j+1} \cdots \tilde{\pi}_{m} \tilde{P}_{m}\right)
$$

where $M$ has the $k$-homotopy-type as $S^{n}$, and $Q_{i}$ has the same $k$-homotopy-type as $X_{i}$. The dimensions may be chosen as in Theorem 1. It is easy to see, using standard theorems on transversality, that it is no loss of generality to assume the base point in each space is a regular value of the maps for which it lies in the range.

We choose $V$ to have the same $k$-type as $S^{p} \subseteq V$, with dimension bigger than that of $M$, and select a differentiable extension map $\tilde{g}$ : $V \rightarrow M$, homotopy-equivalent to $g$ through dimension $k$. We may clearly assume, without loss of generality, that the base point in $M$ lies in $S^{n} \subseteq M$ and is a regular value for $\tilde{g} \mid S^{p}$. The usual density of transverse maps (Thom lemma) assures us that we may also assume that the base point in $M$ is a regular value for $\tilde{g}: V \rightarrow M$, without destroying the fact that it is a regular value for $\tilde{g} \mid S^{p}$.

We now denote

$$
W_{i}=\left(\tilde{P}_{i} \cdot \tilde{g}\right)^{-1} \quad \text { (base point) }
$$

(recall $\left.\tilde{P}_{i}=\tilde{\pi}_{i+1} \cdots \tilde{\pi}_{m} \cdot \tilde{P}_{m}\right)$ and

$$
W=\tilde{g}^{-1} \quad \text { (base point). }
$$

This clearly yields the desired decreasing filtration of framed submanifolds of $V$.

We need only prove (3). Consider the diagram

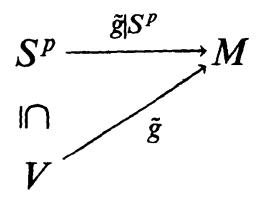

$\tilde{g}$ is an extension of a map homotopic to $g$, and $\left(\tilde{g} \mid S^{p}\right)^{-1}$ (base point) $=$ $\tilde{g}^{-1}$ (base point) $\cap S^{p}$. But $W=\tilde{g}^{-1}$ (base point), completing the proof.

REMARKS. (1) One may clearly also relate the various normal framings on the $W_{i}$ and $W$. 
(2) One may take the intersections of the $W_{l}$ and $S^{p}$, and form a decreasing filtration of subspaces, but I don't know how one can arrange matters so that they are all distinct framed submanifolds. At any rate, the manifold $V$ is naturally related to the homotopy structure, and it is an appropriate place for our filtration to be displayed.

In the way of examples and applications, we first look at the Hopf map $h: S^{3} \rightarrow S^{2}$ from the point of view of Theorem 2 . The first stage in a Postnikov decomposition for $S^{2}$ is

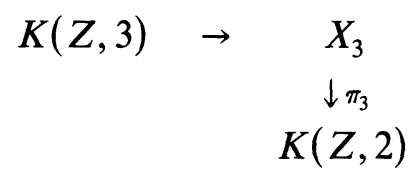

with the $k$-invariant being, up to sign, the square of the generator. To approximate up to dimension 3, we may replace $X_{3}$ by $S^{2}$ and $K(Z, 2)$ by $C P^{2}$. To keep the dimensions increasing, we then replace $S^{2}$ by $S^{2} \times S^{4}$, and mapping $S^{4}$ trivially, we get a differentiable approximation to $\pi_{3}$ as

$$
f: S^{2} \times S^{4} \rightarrow C P^{2} .
$$

For the approximation, through dimension 3, to $h$, we take

$$
h \times 1: S^{3} \times S^{4} \rightarrow S^{2} \times S^{4} .
$$

As in Theorem 2, we must then replace $f$ and $h \times 1$ by close approximations which satisfy the transversality conditions of the theorem.

We then can see easily that $W$ is a circle which actually lies in $S^{3} \subseteq S^{3} \times S^{4}$. There is a single stage in our filtration $W_{2}$ corresponding to the points in $S^{3} \times S^{4}$ which project to a point in $C P^{2}$. This is a three manifold, in $S^{3} \times S^{4}$, containing the circle $W$, which will depend, in general, on the approximations chosen to insure transversality. One may check that it is possible to arrange matters so that $W_{2}$ is the Cartesian product of $S^{1}$ and a compact 2-manifold.

As for potential applications of these methods, we note that there are many algebraic properties of the stable homotopy groups of spheres, which have not been fully analyzed from the purely geometric point of view of framed cobordism. Examples would be filtration in the Adams and other spctral sequences, order, divisibility in the sense of composition product or Toda brackets, etc. There is interest in a geometric understanding of such properties. We signal two potential applications, which will become concrete when the manifolds in Theorem 2 are precisely known.

(a) A Postnikov system determines a filtration of a space, in terms of the fibres of the maps $P_{m}: X \rightarrow X_{m}$ (see [6]). This gives rise to a 1st 
quadrant spectral sequence. In case $X$ is a sphere, in the stable range, the homology sequence contains the stable homotopy of spheres on the $y$-axis, and converges to zero in positive dimensions. Every element in stable homotopy of spheres, in positive dimension, has finite filtration, that is vanishes in some $E^{(r)}, r<\infty$, and this filtration is related to the order of the element (compare [6]). Theorem 2 above offers a geometric interpretation in that an element may only have high filtration if there is a long chain of distinct manifolds $W_{n} \supseteq \cdots \supseteq W_{m}$ from Theorem 2. If one knows enough about these manifolds to assure that a chain of some length is not possible, then one would have a purely geometric interpretation that the filtration of an element is less than some number, as well as a purely geometric interpretation of order.

(b) Suppose $a=b \cdot c$, in the stable homotopy of spheres, with all elements having positive dimension. Then the filtration of $a$, described above, must be less than or equal to the minima of the filtrations of $b$ and c. (See [6].) Therefore, (a) above offers the potential of having a purely geometric understanding of divisibility properties in the stable homotopy of spheres.

In closing, I would like to signal some relevant problems:

(1) Can one specifically calculate these manifolds in explicit, interesting cases?

(2) The methods of Theorem 1, for finding differentiable approximations, clearly apply to finite homotopy resolutions. Is there any meaningful stabilization of these constructions, when the length of the resolution is allowed to go to infinity?

(3) Can one relate the $k$-invariants of the resolution to the manifolds $W_{i}$ and their normal framings?

(4) Can one bring the "induced maps" of [5] into this framework? If the differentiable approximations are fixed in advance, there appear to be difficulties.

(5) What is the effect of a non-trivial (framed) cobordism on these manifolds?

\section{REFERENCES}

[1] J. F. Adams, On the structure and applications of the Steenrod algebra, Comm. Math. Helv., 32 (1958), 180-214.

[2] J. D. Becker and R. E. Schultz, Fixed Point Indices and Left Invariant Framings. Geometric Applications of Homotopy Theory I, Lecture Notes in Math., 657, Springer Verlag (1978).

[3] S. S. Cairns, Smooth approximations to polyhedra, Michigan J. Math., 14 (1967), 305-319. 
[4] H. Gershenson, Relationships between the Adams spectral sequence and Toda's calculations of the stable homotopy groups of spheres, Math. Zeit., 81 (1963), 223-259.

[5] D. W. Kahn, Induced maps for Postnikov systems, Trans. Amer. Math. Soc., 107, No. 3 (1963), 432-450.

[6] The spectral sequence of a Postnikov system, Comm. Math. Helv., 40, 3, (1966), 169-198.

[7] E. Ossa, Lie groups as framed manifolds, Topology, 21, No. 3 (1982), 315-324.

[8] L. Pontrjagin, Smooth manifolds and their applications in homotopy theory, Trudy. Mat. Inst. Steklow, 45, Moscow 1955, Amer. Math. Soc. Translation series 2, vol. 11, (1959).

[9] H. Toda, Composition Methods in Homotopy Groups of Spheres, Princeton U. Press, 1962.

Received September 20, 1982 and in revised form March 16, 1983.

UNIVERSITY OF MINNESOTA

MINNEAPOLIS, MN 55455 


\section{PACIFIC JOURNAL OF MATHEMATICS \\ EDITORS}

Donald BaBBITT (Managing Editor)

University of California

Los Angeles, CA 90024

Hugo RossI

University of Utah

Salt Lake City, UT 84112

C. C. Moore and Arthur Ogus

University of California

Berkeley, CA 94720
J. DugundiI

Department of Mathematics

University of Southern California

Los Angeles, CA 90089-1113

R. FINN and H. SAMELSON

Stanford University

Stanford, CA 94305

\section{ASSOCIATE EDITORS}
R. ARENS
E. F. BECKENBACH
B. H. NeUMANN
F. WOLF
K. YoshidA (1906-1982)

\section{SUPPORTING INSTITUTIONS}

\author{
UNIVERSITY OF ARIZONA \\ UNIVERSITY OF BRITISH COLUMBIA \\ CALIFORNIA INSTITUTE OF TECHNOLOGY \\ UNIVERSITY OF CALIFORNIA \\ MONTANA STATE UNIVERSITY \\ UNIVERSITY OF NEVADA, RENO \\ NEW MEXICO STATE UNIVERSITY \\ OREGON STATE UNIVERSITY
}

\author{
UNIVERSITY OF OREGON \\ UNIVERSITY OF SOUTHERN CALIFORNIA \\ STANFORD UNIVERSITY \\ UNIVERSITY OF HAWAII \\ UNIVERSITY OF TOKYO \\ UNIVERSITY OF UTAH \\ WASHINGTON STATE UNIVERSITY \\ UNIVERSITY OF WASHINGTON
}

The Supporting Institutions listed above contribute to the cost of publication of this Journal, but they are not owners or publishers and have no responsibility for its content or policies.

Mathematical papers intended for publication in the Pacific Journal of Mathematics should be in typed form or offset-reproduced (not dittoed), double spaced with large margins. Please do not use built up fractions in the text of the manuscript. However, you may use them in the displayed equations. Underline Greek letters in red, German in green, and script in blue. The first paragraph must be capable of being used separately as a synopsis of the entire paper. In particular it should contain no bibliographic references. Please propose a heading for the odd numbered pages of less than 35 characters. Manuscripts, in triplicate, may be sent to any one of the editors. Please classify according to the scheme of Math. Reviews, Index to Vol. 39. Supply name and address of author to whom proofs should be sent. All other communications should be addressed to the managing editor, or Elaine Barth, University of California, Los Angeles, California 90024.

There are page-charges associated with articles appearing in the Pacific Journal of Mathematics. These charges are expected to be paid by the author's University, Government Agency or Company. If the author or authors do not have access to such Institutional support these charges are waived. Single authors will receive 50 free reprints; joint authors will receive a total of 100 free reprints. Additional copies may be obtained at cost in multiples of 50 .

The Pacific Journal of Mathematics is issued monthly as of January 1966. Regular subscription rate: $\$ 132.00$ a year (6 Vol., 12 issues). Special rate: $\$ 66.00$ a year to individual members of supporting institutions.

Subscriptions, orders for numbers issued in the last three calendar years, and changes of address should be sent to Pacific Journal of Mathematics, P.O. Box 969, Carmel Valley, CA 93924, U.S.A. Old back numbers obtainable from Kraus Periodicals Co., Route 100, Millwood, NY 10546.

The Pacific Journal of Mathematics ISSN 0030-8730 is published monthly by the Pacific Journal of Mathematics at P.O. Box 969, Carmel Valley, CA 93924. Application to mail at Second-class postage rates is pending at Carmel Valley, California, and additional mailing offices. Postmaster: Send address changes to Pacific Journal of Mathematics, P. O. Box 969, Carmel Valley, CA 93924.

PUBLISHED BY PACIFIC JOURNAL OF MATHEMATICS, A NON-PROFIT CORPORATION

Copyright $\odot 1984$ by Pacific Journal of Mathematics 


\section{Pacific Journal of Mathematics}

\section{Vol. 113, No. $2 \quad$ April, 1984}

Alan Adolphson, On the Dwork trace formula ...................257

Amos Altshuler and Leon Steinberg, Enumeration of the quasisimplicial

3 -spheres and 4-polytopes with eight vertices .................. 269

Kenneth R. Goodearl, Cancellation of low-rank vector bundles .......... 289

Gary Fred Gruenhage, Ernest A. Michael and Yoshio Tanaka, Spaces

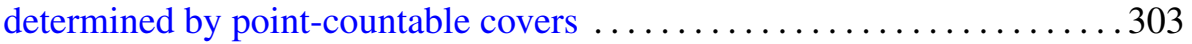

Charles Lemuel Hagopian, Atriodic homogeneous continua .......... 333

David Harbater, Ordinary and supersingular covers in characteristic $p$. . . 349

Domingo Antonio Herrero, Continuity of spectral functions and the lakes

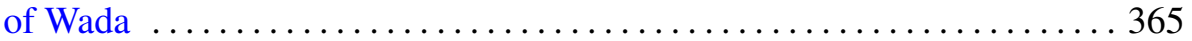

Donald William Kahn, Differentiable approximations to homotopy resolutions and framed cobordism ....................... 373

K. McGovern, On the lifting theory of finite groups of Lie type $\ldots \ldots \ldots . \ldots 383$

C. David (Carl) Minda, The modulus of a doubly connected region and the geodesic curvature-area method ............................. 395

Takuo Miwa, Complexes are spaces with a $\sigma$-almost locally finite base $\ldots . .407$

Ho Kuen Ng, Finitely presented dimension of commutative rings and modules

Roger David Nussbaum, A folk theorem in the spectral theory of

$C_{0}$-semigroups

J. S. Okon, Prime divisors, analytic spread and filtrations

Harold Raymond Parks, Regularity of solutions to elliptic isoperimetric problems

R. Sitaramachandra Rao and M. V. Subba Rao, Transformation formulae for multiple series

Daniel Ruberman, Imbedding punctured lens spaces and connected sums

Uri Srebro, Deficiencies of immersions 